\title{
A study of the capability of the LHAASO experiment to separate primary mass groups samples
}

\section{A. Chiavassa ${ }^{* 1}$ for the LHAASO Collaboration}

${ }^{1}$ Dipartimento di Fisica Università degli Studi di Torino and INFN, Italy

E-mail: andrea.chiavassa@to.infn.it

The LHAASO experiment will operate in the Sichuan province (China) at high altitude (4410 $\mathrm{m}$ a.s.l.) measuring the electromagnetic and muonic EAS components covering a $1 \mathrm{~km}^{2}$ surface with an unprecedented high ratio between the active and the effective areas. The EAS electromagnetic component will be measured by $56351 \mathrm{~m}^{2}$ plastic scintillator detectors and the muonic one by 1221 water cherenkov detectors, $3.4 \mathrm{~m}$ radius and $1.2 \mathrm{~m}$ high each, buried $2.5 \mathrm{~m}$ underneath the ground surface. 24 movable telescopes (each of them made by a $5 \mathrm{~m}^{2}$ spherical mirrors seen by a grid of 32x32 photomultipliers) will be used to detect either the Cherenkov or the Fluorescence light emitted during the shower development in atmosphere.

In this contribution we present the experiment capabilities of separating, using the ratio between the muon and electron numbers at observation level, at least two mass groups in the $10^{14}-10^{17} \mathrm{eV}$ energy range. The study has been performed by mean of a complete EAS simulation for fixed primary energy and fixed zenith angle, using the CORSIKA code and the QSGJetII-04 hadronic interaction model. Therefore current results represent only the first step toward a complete discussion. We will show that an experiment operating at high altitude (i.e. near to shower maximum) will separate, in this energy range, at least two mass groups (i.e. the light and the heavy primaries) with high efficiency.

The 34th International Cosmic Ray Conference,

30 July- 6 August, 2015

The Hague, The Netherlands

\footnotetext{
* Speaker.
} 


\section{Introduction}

The Large High Altitude Air Shower Observatory (LHAASO) project is a new generation instrument, that will be built at 4400 meters altitude in the Sichuan province of China, with the goal of studying with unprecedented sensitivity the spectrum and composition of cosmic rays in the broad energy range between $10^{13}$ and $10^{18} \mathrm{eV}$, as well as to act simultaneously as a wide aperture (one stereo radiant), continuously-operated gamma ray telescope in the energy range between $10^{11}$ and $10^{15} \mathrm{eV}$.

The range of energy for cosmic ray studies in LHAASO contains the most prominent structure in the cosmic ray energy spectrum, the so called knee, at $E \sim 3 \times 10^{15} \mathrm{eV}$, where the index of the power-law spectrum steepens from $\gamma \sim 2.7$ to $\gamma \sim 3.1$. There are clear indications that the cosmic ray composition is evolving around the knee energy toward a mixture where heavy nuclei are more abundant[1]. The physical origin of the knee remains controversial, and more precise measurements of the evolution of the energy spectrum and of the mass composition in this region are mandatory to discriminate about existing models. At higher energy, studies of the energy spectrum and mass composition of cosmic rays in the range $10^{16}-10^{18} \mathrm{eV}$ have given indications for the existence of other structures, associated to the steepening of the flux of different nuclei[2], and perhaps to the appearance of extragalactic lighter nuclei[3].

In this contribution we discuss the potential of an experiment operating at $4410 \mathrm{~m}$ a.s.l. to separate primary mass group samples by means of the experimental observable obtained by the ratio between the electron and muon numbers at observation level. This work is a preliminary study performed simulating EAS generated at fixed energies, fixed zenith angle and counting all the particles arriving at observation level, i.e. no detector layout is taken into account. Therefore the results we will discuss represent the ideal case of an experiment without detection and reconstruction errors and $100 \%$ coverage. We must also consider that fluctuations are minimized simulating EAS at fixed energies instead of extracting the primary energy on a power law spectrum. All these effects will be taken into account in a future complete EAS and detector simulation that is currently under development.

\section{The Experiment}

The layout of the experiment includes different types of detectors:

- a $1 \mathrm{~km}^{2}$ array (LHAASO-KM2A[4]), including 5635 plastic scintillator detectors, $1 \mathrm{~m}^{2}$ surface each separated by $15 \mathrm{~m}$ spacing, for electromagnetic particle detection.

- An overlapping $1 \mathrm{~km}^{2}$ array of $1221,36 \mathrm{~m}^{2}$ underground water Cherenkov tanks, with $30 \mathrm{~m}$ spacing, for muon detection (total sensitive area $4 \times 10^{4} \mathrm{~m}^{2}$ ).

- A close-packed, surface water Cherenkov detector facility with a total area of $9 \times 10^{4} \mathrm{~m}^{2}$ (LHAASO-WCDA)[5], four times that of HAWC[6].

- 24 wide field-of-view air Cherenkov (and fluorescence) telescopes (LHAASO-WFCTA)[7].

- 452 close-packed burst detectors, located near the center of the array, for detection of high energy secondary particles in the shower core region (LHAASO-SCDA). 
LHAASO will be located at high altitude (4410 $m$ a.s.l., $\left.600 \mathrm{gcm}^{-2}, 29^{\circ} 21^{\prime} 31^{\prime \prime} \mathrm{N}, 100^{\circ} 08^{\prime} 15^{\prime \prime} E\right)$ in the Daochen site, Sichuan province, P.R. China. The start of data taking is expected in 2-3 years. The completion of installation in 5-6 years.

It is therefore clear that LHAASO will operate over a large surface having a quite high coverage: being the ratio between the active and the effective areas for the electromagnetic component equal to $\sim 5 \times 10^{3} / 10^{6}=5 \times 10^{-3}$, i.e. a coverage similar to the KASCADE experiment[8] $\left(\sim 5 \times 10^{2} / 4 \times 10^{4}=10^{-2}\right)$ spread in a 25 times wider area. The same is true also for the muon EAS component: the LHAASO coverage $\left(\sim 5 \times 10^{4} / 10^{6}=5 \times 10^{-2}\right)$ will be even higher than the one of the KASCADE array $\left(6 \times 10^{2} / 4 \times 10^{4}=1.5 \times 10^{-2}\right)$. We therefore expect to have very precise measurements of the EAS components

\section{The simulation}

The simulation used to obtain the results discussed in this contribution has been performed with the CORSIKA code[9] using the QGSJetII-04[10] hadronic interaction model at high energies $(E>200 \mathrm{GeV})$ and FLUKA[11] at lower energies.The CORSIKA code was run with the EGS4 option for the electromagnetic component (i.e. electron and photon are fully simulated), no thinning option was adopted, i.e. all the particles in the shower are followed until very low energies $(3 \mathrm{MeV}$ for electron and photons, $100 \mathrm{MeV}$ for muon and hadrons). The observation level is set at the LHAASO height: $4400 \mathrm{~m}$ a.s.l., all events are simulated in the vertical direction $\left(\theta=0^{\circ}\right)$.

With these options the simulation code becomes, at high energies, quite heavy in terms of both running time and disk space allocated. EASs have been simulated with equal number of events for $\mathrm{H}, \mathrm{He}, \mathrm{C}$ and $\mathrm{Fe}$ primaries, the energies simulated and the number of showers generated are reported in table 1

\begin{tabular}{|l|c|c|c|c|c|c|}
\hline Energy $(\mathrm{eV})$ & $10^{15}$ & $5 \times 10^{15}$ & $10^{16}$ & $5 \times 10^{16}$ & $10^{17}$ & $5 \times 10^{17}$ \\
\hline Events & 250 & 250 & 150 & 50 & 25 & 5 \\
\hline
\end{tabular}

Table 1: Primary energies of the simulated EAS and number of events generated for each primary particle $(\mathrm{H}, \mathrm{He}, \mathrm{C}, \mathrm{Fe})$

\section{Results}

Figures 1, 2 and 3 show the electrons, muon and pion lateral distributions at observation level. We can see that the electron density goes below 1 particle $/ \mathrm{m}^{2}$ at $\sim 200 \mathrm{~m}$ and $\sim 400 \mathrm{~m}$ respectively for $5 \times 10^{15}$ and $5 \times 10^{16} \mathrm{eV}$ primary energy and all primaries. Being one square meter the surface of the unshielded detectors we can expect that these distances will coincide with the radius containing almost all the fired detectors in an event. While from the highest energies $\left(5 \times 10^{17} \mathrm{eV}\right)$ we can see that the expected particle density goes beyond $10^{4}$ particles (i.e. the detector saturation level) at $50 \mathrm{~m}$ from the shower core. With the expected dynamic range detector saturation will therefore affect, even for the most energetic showers, few detectors located very near to the shower core.

Muon lateral distribution can only be used to have a first idea of the particle density hitting the detectors as the muon energy threshold to cross the overburden covering the water tanks is not 


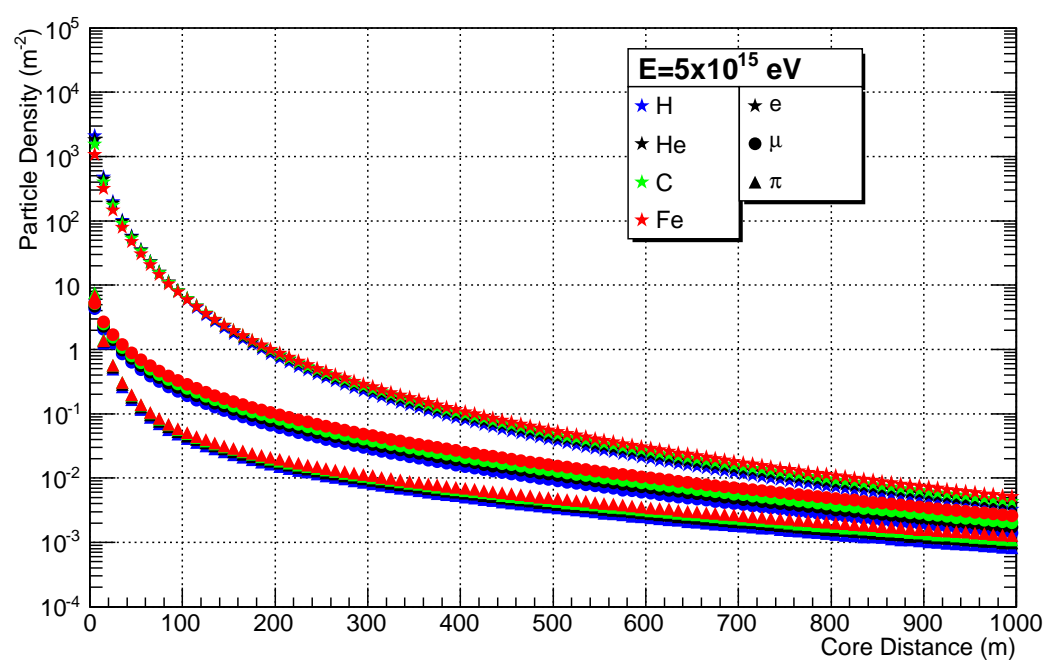

Figure 1: Lateral distributions of the electron, muon and pion EAS components at $4400 \mathrm{~m}$ a.s.l. obtained simulating vertical showers generated by primaries of energy $E=5 \times 10^{15} \mathrm{eV}$.

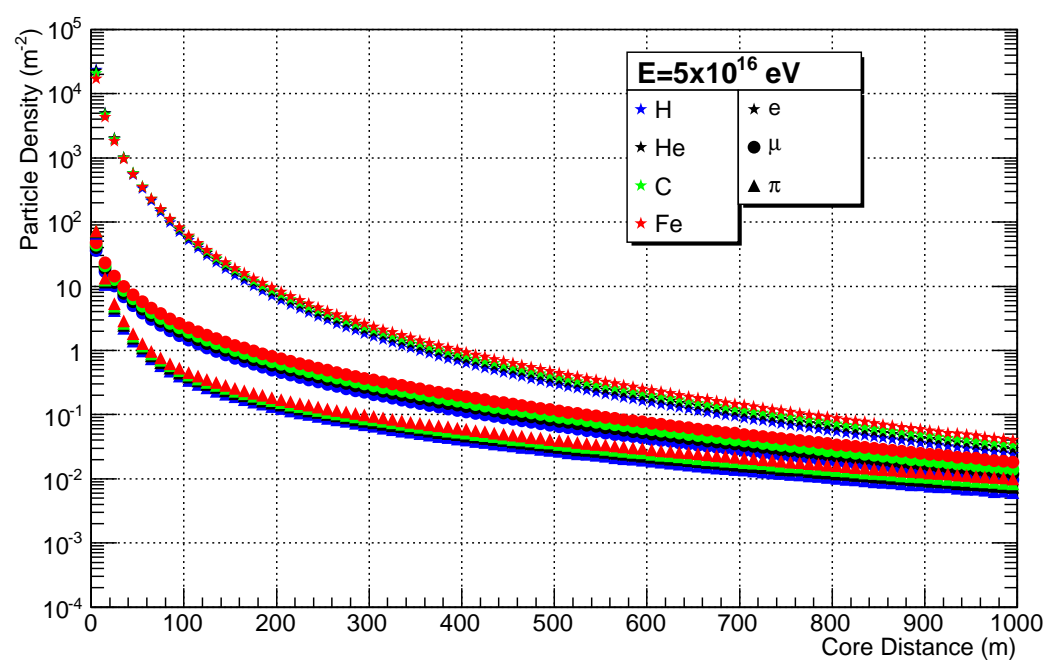

Figure 2: Lateral distributions of the electron, muon and pion EAS components at $4400 \mathrm{~m}$ a.s.l. obtained simulating vertical showers generated by primaries of energy $E=5 \times 10^{16} \mathrm{eV}$.

taken into account. Being the muon detectors surface $36 \mathrm{~m}^{2}$ we can expect that one EAS particle hits a muon detector if their density is greater than $0.027 \mathrm{~m}^{-2}$ therefore we can expect that muon detectors can give signals at larger distances from the core compared to the electron detectors.

We can also notice that the electron lateral distributions of EAS generated by light primaries are higher than those expected for EAS generated by heavy primaries near to shower core, becoming smaller at large core distances. While the muon densities expected for heavy primaries are 


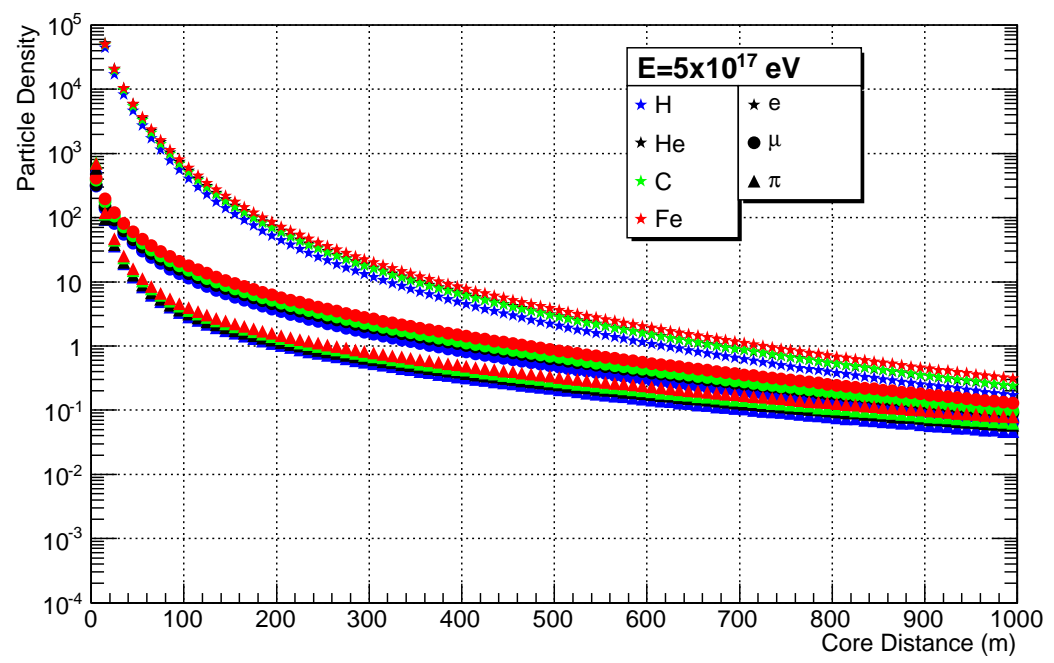

Figure 3: Lateral distributions of the electron, muon and pion EAS components at $4400 \mathrm{~m}$ a.s.l. obtained simulating vertical showers generated by primaries of energy $E=5 \times 10^{17} \mathrm{eV}$.

larger than those for light primaries at all shower core distances.

A first attempt to evaluate the possibilities to separate events in at least two mass groups can be obtained studying the ratio between the total muon and electron numbers at observation level. For each simulated EAS we therefore count the electron and muon numbers (without taking into account any energy threshold) and calculate their ratio:

$$
Y=\frac{\log N_{\mu}\left(\theta=0^{\circ}\right)}{\log N_{e}\left(\theta=0^{\circ}\right)}
$$

the result is shown in figure 4. Being interested in a event by event classification the error bars represent the RMS of the distributions. Iron generated shower are clearly separated from all other primaries, therefore we have promising perspectives to identify heavy and light primaries with good precision and with small contaminations. While the mean values of $\mathrm{H}$ and $\mathrm{He}$ and of $\mathrm{He}$ and $\mathrm{C}$ generated showers are separated by less than one standard deviation, therefore, with this simple approach, we cannot expect to perform a good separation of individual elements. We must always keep in mind that this plot is only showing a very simple approach using two of all the detectors that will be installed in the LHAASO experiment, detailed studies will be performed (simulating showers on a power spectrum, at all zenith angles, including the detector layout and performances) in the near future taking advantage of all the possibilities of the experiment.

\section{Acknowledgement}

This work is supported in China by NSFC (NO.11205165, NO. 11375210, No.11375224, No.11405181, No.11475190), the Chinese Academy of Science, Institute of High Energy Physics, the Key Laboratory of Particle Astrophysics, CAS and in Italy by the istituto nazionale di fisica nucleare (INFN). 


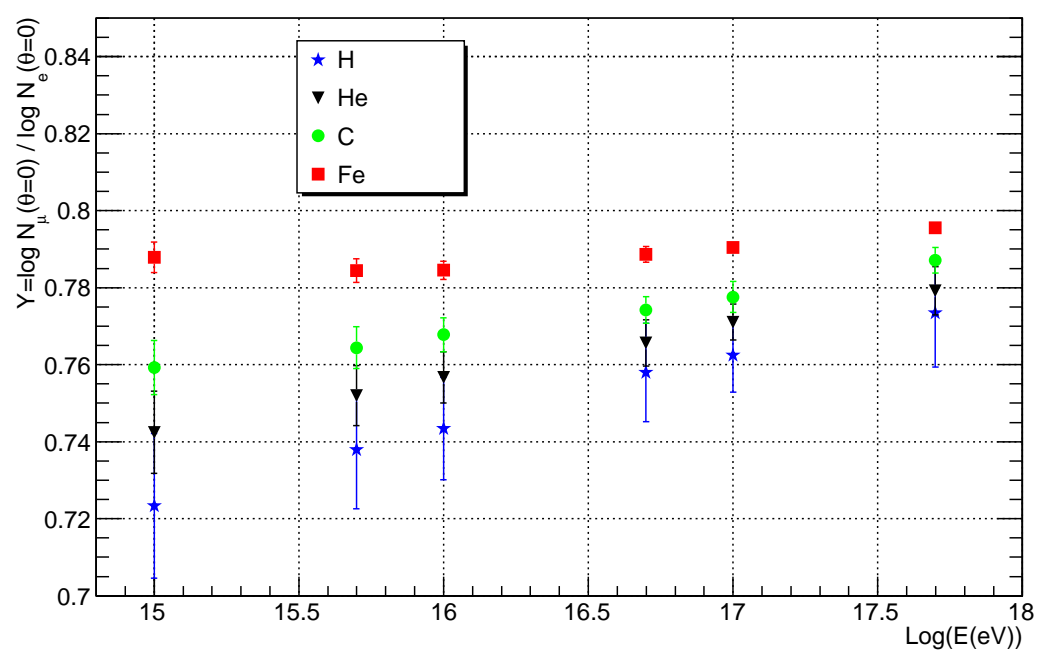

Figure 4: Y values obtained by a full EAS simulation. Array layout and detection errors are not taken into account. These values represent the ideal performances of a $100 \%$ coverage experiment with no reconstruction errors.

\section{References}

[1] T. Antoni et al., Astropart. Phys. 24, 1 (2005).

[2] W.D. Apel et al., Phys. Rev. Lett. 107, 171104 (2011).

[3] W.D. Apel et al., Phys. Rev. D 87, 081101(R) (2013)

[4] J. Zhao et al., Chinese Physics C 38, 036002 (2014).

[5] H. C. Li et al., Chinese Physics C 38, 016002 (2014).

[6] A. U. Abeysekara et al., Astropart. Phys. 50-52 (2013), 26-32.

[7] S. S. Zhang et al., Nucl Instr. \& Meth. A 629, 57 (2011).

[8] T. Antoni et al., Nucl. Instr. \& Meth. A 513, 429 (2003).

[9] D. Heck et al., Report FZKA 6019, Forschungszentrum Karlsruhe (1998).

[10] S. Ostapchenko, Phys. Rev. D 83, 014018 (2011).

[11] A. Fassò et al., Report CERN-2005-10, INFN/TC-05/11, SLAC-R-773 (2005). 